IJMMS 29:1 (2002) 55-61

PII. S0161171202011134

http://ijmms.hindawi.com

(c) Hindawi Publishing Corp.

\title{
A NOTE ON HOMOMORPHISMS OF HILBERT ALGEBRAS
}

\author{
SERGIO CELANI
}

Received 21 November 2000 and in revised form 14 May 2001

\begin{abstract}
We give a representation theorem for Hilbert algebras by means of ordered sets and characterize the homomorphisms of Hilbert algebras in terms of applications defined between the sets of all irreducible deductive systems of the associated algebras. For this purpose we introduce the notion of order-ideal in a Hilbert algebra and we prove a separation theorem. We also define the concept of semi-homomorphism as a generalization of the similar notion of Boolean algebras and we study its relation with the homomorphism and with the deductive systems.
\end{abstract}

2000 Mathematics Subject Classification: 08A30.

1. Introduction and preliminaries. Hilbert algebras correspond to the algebraic counterpart of the implicative fragment of Intuitionistic Propositional Logic. For the general development of Hilbert algebras, the notion of deductive system (also called implicative filters) plays an important role. For example, it is known that the set of all deductive systems of a Hilbert algebra is a distributive lattice isomorphic to the lattice of congruences (see [2, 4]). In [4], Diego proved that every Hilbert algebra can be represented as a subalgebra of the Hilbert algebra of the open subsets of a suitable $T_{0}$ topological space. Our central aim is to prove a new representation theorem for Hilbert algebras by means of ordered sets and give some results relating homomorphisms of Hilbert algebras to deductive systems and a weaker version of homomorphisms.

In Section 2, we start recalling the notion of deductive system and irreducible deductive system. We prove a theorem of separation, and a representation theorem for Hilbert algebras by means of ordered sets. In Section 3, we introduce the notion of semi-homomorphism between Hilbert algebras. We prove a characterization of the semi-homomorphism by means of deductive systems and we prove a characterization of the homomorphism by means of irreducible deductive systems. To conclude, we deal with the study of injective and surjective homomorphism of Hilbert algebras.

We note that Hilbert algebras are dual to positive implicative BCK-algebras. This duality was first noted in [3], and fully proved in [1,5]. Thus, the results given in this note can be applied straightly to the positive implicative BCK-algebras.

DeFinITION 1.1. A Hilbert algebra is an algebra $\langle A, \rightarrow, 1\rangle$ where $A$ is a nonempty set, $\rightarrow$ is a binary operation on $A, 1$ is an element of $A$ such that the following conditions are satisfied for every $a, b, c \in A$ :

(H1) $a \rightarrow(b \rightarrow a)=1$.

(H2) $(a \rightarrow(b \rightarrow c)) \rightarrow((a \rightarrow b) \rightarrow(a \rightarrow c))=1$.

(H3) If $a \rightarrow b=1$ and $b \rightarrow a=1$, then $a=b$. 
Let $A$ be a Hilbert algebra. It is known that the relation $\leq$ defined in $A$ by $a \leq b$ if and only if $a \rightarrow b=1$ is a partial order on $A$ where 1 is the greatest element of $A$ with respect to this ordering.

Proposition 1.2. Let $A$ be a Hilbert algebra. Then the following properties are valid for any $a, b, c \in A$ :

(1) $a \leq b \rightarrow a$.

(2) $a \rightarrow 1=1$.

(3) $1 \rightarrow a=a$.

(4) $a \rightarrow(b \rightarrow c)=b \rightarrow(a \rightarrow c)$.

(5) $a \rightarrow(b \rightarrow c)=(a \rightarrow b) \rightarrow(a \rightarrow c)$.

(6) If $a \leq b$, then $c \rightarrow a \leq c \rightarrow b$ and $b \rightarrow c \leq a \rightarrow c$.

Proof. See [2] or [4].

\section{Representation theorem}

Definition 2.1. Let $A$ be a Hilbert algebra. A deductive system of $A$ is a subset $D$ of $A$ such that

(D1) $1 \in D$,

(D2) if $a, a \rightarrow b \in D$, then $b \in D$.

Let $A$ be a Hilbert algebra. The set of all deductive systems of $A$ is denoted by $\operatorname{Ds}(A)$. It is known that $\operatorname{Ds}(A)$ is a distributive lattice (see [4]). If $X \subseteq A$, then the deductive system generated by $X$, in symbols $D(X)$, is the set (see [2] or [4])

$$
D(X)=\left\{a \in A: \exists\left\{x_{0}, x_{1}, \ldots, x_{n}\right\} \subseteq X: x_{0} \longrightarrow\left(x_{1} \longrightarrow \cdots\left(x_{n} \longrightarrow a\right) \cdots\right)=1\right\}
$$

DeFinition 2.2. Let $A$ be a Hilbert algebra. Let $D \in \operatorname{Ds}(A)$. Then we say that

(1) $D$ is irreducible if and only if for any $D_{1}, D_{2} \in \operatorname{Ds}(A)$ such that $D=D_{1} \cap D_{2}$, then $D=D_{1}$ or $D=D_{2}$.

(2) $D$ is completely irreducible if and only if for any family $H=\left\{D_{i}: i \in I\right\} \subseteq \operatorname{Ds}(A)$ such that $D=\bigcap\left\{D_{i}: i \in I\right\}$, then $D=D_{i}$ for some $i \in I$.

We denote the set of all irreducible and the set of all completely irreducible deductive systems of $A$, by $X(A)$ and $X_{c}(A)$, respectively.

The following results are known (see [2, 4]).

THEOREM 2.3. Let $A$ be a Hilbert algebra. Then

(1) $D \in X(A)$ if and only if for every $a, b \notin D$ there exists $c \notin D$ such that $a \leq c$ and $b \leq c$.

(2) $D \in X_{c}(A)$ if and only if there exists $c \notin D$ such that $a \rightarrow c \in D$ for every $a \notin D$.

(3) For all $a, b \in A$ if $a \ddagger b$ there exists $D \in X_{\mathcal{C}}(A)$ such that $a \in D$ and $b \notin D$.

The following result gives another characterization of irreducible deductive systems in a Hilbert algebra.

LEMmA 2.4. Let $A$ be a Hilbert algebra. Let $D \in \operatorname{Ds}(A)$. Then $D$ is irreducible if and only if for every $a, b \notin D$ there exists $c \notin D$ such that $a \rightarrow c, b \rightarrow c \in D$. 
Proof. The direction $(\Rightarrow$ ) follows by assertion 1 of Theorem 2.3.

$(\Leftarrow)$. Suppose that $D_{1}, D_{2} \in \operatorname{Ds}(A)$ such that $D=D_{1} \cap D_{2}, D \neq D_{1}$ and $D \neq D_{2}$. Then there exist $a \in D_{1}-D$ and $b \in D_{2}-D$. Then, by assumption, there exists $c \notin D$ such that $a \rightarrow c \in D$ and $b \rightarrow c \in D$. So, $a, a \rightarrow c \in D_{1}$ and as $D_{1} \in \operatorname{Ds}(A)$, then $c \in D_{1}$. Similarly, $c \in D_{2}$. Thus, $c \in D_{1} \cap D_{2}=D$, which is a contradiction.

DeFinITION 2.5. Let $A$ be a Hilbert algebra. A subset $I$ of $A$ is called an order-ideal of $A$ if

(ID1) If $b \in I$ and $a \leq b$, then $a \in I$.

(ID2) If $a, b \in I$ then there exists $c \in I$ such that $a \leq c$ and $b \leq c$.

The set of all order-ideals of $A$ will be denoted by $\operatorname{Id}(A)$.

Now, we prove the mentioned separation theorem.

THEOREM 2.6. Let $A$ be a Hilbert algebra. Let $D \in \operatorname{Ds}(A)$ and $I \in \operatorname{Id}(A)$ such that $D \cap I=\varnothing$. Then there exists $P \in X(A)$ such that $D \subseteq P$ and $P \cap I=\varnothing$.

Proof. Consider the following subset of $\operatorname{Ds}(A)$ :

$$
\mathscr{F}=\{H \in \operatorname{Ds}(A): D \subseteq H \text { and } H \cap I=\varnothing\} .
$$

Since $D \in \mathscr{F}$, then $\mathscr{F} \neq \varnothing$. It is clear that the union of a chain of elements of $\mathscr{F}$ is also in $\mathscr{F}$. So, by Zorn's lemma, there exists a deductive systems $P$ maximal in $\mathscr{F}$. We prove that $P \in X(A)$. Let $a, b \notin P$ and consider the deductive systems $P_{a}=D(P \cup\{a\})$ and $P_{b}=D(P \cup\{b\})$. Clearly, $P \subset P_{a} \cap P_{b}$. Then, $P_{a}, P_{b} \notin \mathscr{F}$. Thus, $P_{a} \cap I \neq \varnothing$ and $P_{a} \cap I \neq \varnothing$. It follows that there exist $x, y \in I$ such that $a \rightarrow x \in P$ and $b \rightarrow y \in P$. Since $I$ is an order-ideal, there exists $c \in I$ such that $x \leq c$ and $y \leq c$. So, $a \rightarrow x \leq a \rightarrow c \in P$ and $b \rightarrow y \leq b \rightarrow c \in P$. Therefore, by Lemma 2.4, we conclude that $P \in X(A)$.

Let $\langle X, \leq\rangle$ be a poset. For $Y \subseteq X$, we put $[Y)=\{x \in X: y \leq x$ for some $y \in Y\}$. A subset $Y$ of $X$ is increasing if $Y=[Y)$. The sets of all increasing subsets of $X$ will be denoted by $\mathscr{P}_{i}(X)$.

LEMMA 2.7. Let $\langle X, \leq\rangle$ be a poset. Then $\left\langle\mathscr{P}_{i}(X), \Rightarrow, X\right\rangle$ is a Hilbert algebra where the implication $\Rightarrow$ is defined by

$$
U \Longrightarrow V=\{x \in X:[x) \cap U \subseteq V\},
$$

for $U, V \in \mathscr{P}_{i}(X)$.

Proof. The proof is immediate.

Let $A$ be a Hilbert algebra. Consider the poset $\langle X(A), \subseteq\rangle$ and consider the mapping

$$
\beta: A \longrightarrow \mathscr{P}_{i}(X(A))
$$

defined by

$$
\beta(a)=\{P \in X(A): a \in P\} .
$$

THEOREM 2.8 (representation theorem). Let $A$ be a Hilbert algebra. Then, $A$ is isomorphic to the subalgebra $\beta(A)=\{\beta(a): a \in A\}$ of $\mathscr{P}_{i}(X(A))$. 
Proof. It is clear that the map $\beta: A \rightarrow \mathscr{P}_{i}(X(A))$ is injective. We prove that $\beta(a \rightarrow$ $b)=\beta(a) \Rightarrow \beta(b)$. Let $P, Q \in X(A)$ and $a, b \in A$ such that $a \rightarrow b \in P$. If $Q \in[P) \cap \beta(a)$, then $Q \in \beta(b)$, that is, $b \in Q$.

Suppose that $a \rightarrow b \notin P$. Consider the deductive systems $P_{a}=D(P \cup\{a\})$. Since $b \notin P_{a}$, then there exists $Q \in X(A)$ such that $P \subseteq Q, a \in Q$ and $b \notin Q$. Thus, $P \notin$ $\beta(a) \Rightarrow \beta(b)$.

3. Homomorphisms of Hilbert algebras. The purpose of this section is to study the homomorphism between Hilbert algebras.

DEFINITION 3.1. Let $A$ and $B$ be Hilbert algebras. A semi-homomorphism is a mapping $h: A \rightarrow B$ such that

(SH1) $h(1)=1$,

(SH2) $h(a \rightarrow b) \leq h(a) \rightarrow h(b)$, for every $a, b \in A$.

THEOREM 3.2. Let $A$ and $B$ be Hilbert algebras. Let $h: A \rightarrow B$ be a mapping. Then the following conditions are equivalent:

(1) $h$ is a semi-homomorphism

(2) $h^{-1}(D) \in \operatorname{Ds}(A)$ for all $D \in \operatorname{Ds}(B)$.

Proof. (1) $\Rightarrow(2)$. Let $D \in \operatorname{Ds}(B)$. By condition (SH1), $1 \in h^{-1}(D)$. If $a, a \rightarrow b \in$ $h^{-1}(D)$, then $h(a), h(a \rightarrow b) \leq h(a) \rightarrow h(b) \in D$. Since $D$ is a deductive system, then $h(b) \in D$, that is, $b \in h^{-1}(D)$. Thus, $h^{-1}(D) \in \operatorname{Ds}(A)$.

(2) $\Rightarrow(1)$. Suppose that $h^{-1}(D) \in \operatorname{Ds}(A)$ for all $D \in \operatorname{Ds}(B)$. If $h(1) \neq 1$, then there exists $D \in \operatorname{Ds}(B)$ such that $h(1) \notin D$, that is, $1 \notin h^{-1}(D)$, which is impossible. Thus, $h(1)=1$. Suppose that $h(a \rightarrow b) \nless h(a) \rightarrow h(b)$. Then, there exists $P \in X(B)$ such that $h(a \rightarrow b) \in P$ and $h(a) \rightarrow h(b) \notin P$. Consider the deductive system $D(P \cup h(a))$. Since $h(b) \notin D(P \cup h(a))$, there exists $Q \in X(A)$ such that $P \subseteq Q, h(a) \in Q$ and $h(b) \notin Q$. As $a \rightarrow b \in h^{-1}(P) \subseteq h^{-1}(Q)$ and $a \in h^{-1}(Q)$, then $b \in h^{-1}(Q)$, which is a contradiction. Therefore, $h(a \rightarrow b) \leq h(a) \rightarrow h(b)$.

Let $A$ be a Hilbert algebra. The set complement of a subset $P$ of $A$ is denoted by $P^{c}$.

Let $A$ and $B$ be Hilbert algebras. Let $h: A \rightarrow B$ be a homomorphism. It is easy to see that, for each $P \in X(A)$, the subset of $B$

$$
\left(h\left(P^{c}\right)\right]=\{y \in B: y \leq h(p) \text { for some } p \notin P\}
$$

is an order-ideal of $B$. This fact will be used in the following results.

THEOREM 3.3. Let $A$ and $B$ be Hilbert algebras. Let $h: A \rightarrow B$ be a semihomomorphism. Then the following conditions are equivalent:

(1) $h$ is a homomorphism.

(2) For every $(P, Q) \in X(B) \times X(A)$ such that $h^{-1}(P) \subseteq Q$ there exists $D \in X(B)$ such that $P \subseteq D$ and $h^{-1}(D)=Q$.

Proof. (1) $\Rightarrow(2)$. Suppose that $h: A \rightarrow B$ is a homomorphism. Let $(P, Q) \in X(B) \times$ $X(A)$ such that $h^{-1}(P) \subseteq Q$. Consider the deductive system $D(P \cup h(Q))$. Since $Q \in$ $X(A)$ then $\left(h\left(Q^{c}\right)\right] \in \operatorname{Id}(B)$. We prove that

$$
D(P \cup h(Q)) \cap\left(h\left(Q^{c}\right)\right]=\varnothing .
$$


Suppose the contrary. Then there exists $p \in P, q \in Q$, and $a \notin Q$ such that $p \rightarrow(h(q) \rightarrow$ $h(a))=1$. Since $h$ is a homomorphism, then $p \rightarrow h(q \rightarrow a)=1 \in P$. So, $h(q \rightarrow a) \in P$ and this implies that $q \rightarrow a \in h^{-1}(P) \subseteq Q$. Thus, $a \in Q$, which is a contradiction. Therefore, $D(P \cup h(Q)) \cap\left(h\left(Q^{c}\right)\right]=\varnothing$. By Theorem 2.6, there exists $D \in X(B)$ such that $P \subseteq D$ and $h^{-1}(D)=Q$.

(2) $\Rightarrow$ (1). Let $a, b \in A$ and assume that $h(a) \rightarrow h(b) \not h(a \rightarrow b)$. Then there exists $P \in$ $X(B)$ such that $h(a) \rightarrow h(b) \in P$ and $a \rightarrow b \notin h^{-1}(P)$. Since $h$ is a semi-homomorphism, by Theorem 3.2, $h^{-1}(P) \in \operatorname{Ds}(A)$. So, there exists $Q \in X(A)$ such that $h^{-1}(P) \subseteq Q, a \in$ $Q$ and $b \notin Q$. By assumption, there exists $D \in X(B)$ such that $P \subseteq D$ and $h^{-1}(D)=Q$. But since $h(a) \rightarrow h(b) \in P \subseteq D$ and $h(a) \in D$, we get that $h(b) \in D$, which is a contradiction. We conclude that $h$ is a homomorphism.

Let $A$ and $B$ be Hilbert algebras and let $h: A \rightarrow B$ be a homomorphism. Consider the subset of $X(B)$

$$
X_{h}(B)=\left\{P \in X(B): h^{-1}(P) \in X(A)\right\} .
$$

Consider the partial mapping

$$
X(h): X_{h}(B) \longrightarrow X(A)
$$

defined by

$$
X(h)(P)=h^{-1}(P),
$$

with $P \in X_{h}(B)$.

THEOREM 3.4. Let $A$ and $B$ be Hilbert algebras and let $h: A \rightarrow B$ be a homomorphism. Then

(1) $h$ is injective if and only if $X(h)$ is surjective.

(2) $h$ is surjective if and only if $X(h)$ is injective with $X_{h}(B)=X(B)$.

Proof. (1) $(\Rightarrow)$ Let $P \in X(A)$. Consider the deductive system $D(h(P))$. We prove that

$$
D(h(P)) \cap\left(h\left(P^{c}\right)\right]=\varnothing .
$$

Suppose the contrary case. Then there exists $p \in P$ and $a \notin P$ such that $h(p) \rightarrow$ $h(a)=1$. Since $h$ is homomorphism, $h(p \rightarrow a)=1=h(1)$, and as $h$ is injective, we get $p \rightarrow a=1$. So, $a \in P$, which is impossible. Thus, by Theorem 2.6 , there exists $Q \in X(B)$ such that $h(P) \subseteq Q$ and $h\left(P^{c}\right) \cap Q=\varnothing$, that is, $h^{-1}(Q)=P$. Therefore, $X(h)$ is surjective.

$(\Leftarrow)$ Let $a, b \in A$. Suppose that $a \npreceq b$. Then there exists $P \in X(A)$ such that $a \in P$ and $b \notin P$. By assumption, there exists $Q \in X_{h}(B) \subseteq X(B)$ such that $P=h^{-1}(Q)$. Then, $h(a) \in Q$ and $h(b) \notin Q$. It follows that $h(a) \not h(b)$, which implies that $h$ is injective.

$(2)(\Rightarrow)$ First, we prove that $X_{h}(B)=X(B)$. Let $Q \in X(B)$. Since $h$ is a homomorphism, $h^{-1}(Q) \in \operatorname{Ds}(A)$. Let $a, b \in A$ such that $h(a), h(b) \notin Q$. Then there exists $c \notin Q$ such that $h(a) \leq c$ and $h(b) \leq c$. Since $h$ is onto, there exists $d \in B$ such that $c=h(d)$. So, $h(a) \leq h(d)$ and $h(b) \leq h(d)$ with $d \notin h^{-1}(Q)$. Thus, $h^{-1}(Q) \in X(A)$ and consequently $X_{h}(B)=X(B)$. 
Let $P, Q \in X(B)$ such that $h^{-1}(P) \subseteq h^{-1}(Q)$. Then it is easy to check that $P \subseteq Q$. So, $X(h)$ is an order-isomorphism. Thus, $X(h)$ is injective.

$(\Leftarrow)$ Let $b \in B$ and assume that $b \notin h(A)=\{h(a): a \in A\}$. Consider the deductive system $D(b)$ and the deductive system $D\left(h\left(h^{-1}(D(b))\right)\right)$. We prove that

$$
D\left(h\left(h^{-1}(D(b))\right)\right) \cap(b]=\varnothing .
$$

Suppose the contrary. Then there exists $h(x) \in D(b)$ such that $h(x) \leq b$. It follows that $b=h(x)$, which is a contradiction. So, by Theorem 2.6, there exists $P \in X(B)$ such that $h\left(h^{-1}(D(b))\right) \subseteq P$ and $b \notin P$. We prove now that

$$
\left(P^{c} \cap h(A)\right]=\left\{y \in B: y \leq x \text { for some } x \in P^{c} \cap h(A)\right\}
$$

is an order-ideal of $B$. It is enough to prove that if $x, y \in P^{c} \cap h(A)$, then there exists $c \in P^{c} \cap h(A)$ such that $x \leq c$ and $y \leq c$. Let $x, y \in P^{c} \cap h(A)$. Then $x=h(a)$ and $y=h(b)$ for some $a, b \in A$. Since $a, b \notin h^{-1}(P)$ and by assumption, $h^{-1}(P) \in X(B)$, then there exists $c \notin h^{-1}(P)$ such that $a \leq c$ and $b \leq c$. So, $x \leq h(c)$ and $y \leq h(c)$. Thus, $\left(P^{c} \cap h(A)\right]$ is an order-ideal of $B$.

We prove that

$$
D(P \cap h(A) \cup\{b\}) \cap\left(P^{c} \cap h(A)\right]=\varnothing .
$$

Assume the contrary, then there exists $a \in D(P \cap h(A) \cup\{b\}) \cap\left(P^{c} \cap h(A)\right], p=h(q) \in$ $P \cap h(A)$, and $d=h(z) \in P^{c} \cap h(A)$ such that $b \rightarrow(h(q) \rightarrow a)=1$ and $a \leq h(z)$. Thus, $h(q \rightarrow z) \in D(b)$. It follows that $q \rightarrow z \in h^{-1}(D(b)) \subseteq h^{-1}(P)$. Since $q \in P$, then $z \in h^{-1}(P)$, that is, $h(z)=d \in P$, which is absurd. Thus, (3.9) is valid. By Theorem 2.6, there exists $Q \in X(B)$ such that $Q \cap h(A)=P \cap h(A)$ and $b \in Q$. So, $h^{-1}(Q)=h^{-1}(P)$ and as $X(h)$ is injective we conclude that $Q=P$. Thus, $b \in P$, which is a contradiction. So $b \in h(A)$ and therefore $h$ is surjective.

4. Conclusions. In this note, we give a representation theorem for Hilbert algebras and we give several results relating homomorphism of Hilbert algebras to deductive systems and semi-homomorphisms. One of the referees pointed out that these results can help to find a full duality for Hilbert algebras. This problem was one of the motivations to study the representation for Hilbert algebras. Unfortunately, we cannot give a full duality. However, using the results of this note, we have given a duality for implicative semilattice (or positive implicative BCK-algebras with condition (S) [6]). These results will be presented in a future paper.

AcKNowledgement. I would like to thank the referees for the comments and suggestions on the presentation of this note.

\section{REFERENCES}

[1] C. Bărbăcioru, Positive implicative BCK-algebras, Math. Japon. 38 (1993), no. 3, 513-520.

[2] D. Buşneag, On the maximal deductive systems of a bounded Hilbert algebra, Bull. Math. Soc. Sci. Math. R. S. Roumanie (N.S.) 31(79) (1987), no. 1, 9-21.

[3] W. H. Cornish, On positive implicative BCK-algebras, Math. Sem. Notes Kobe Univ. 8 (1980), no. 3, 455-468. 
[4] A. Diego, Sur les algèbres de Hilbert, Collection de Logique Mathématique, Sér. A, Fasc, vol. 21, Gauthier-Villars, Paris, 1966 (French), translated from Spanish by Luisa Iturrioz.

[5] M. Kondo, Hilbert algebras are dual isomorphic to positive implicative BCK-algebras, Math. Japon. 49 (1999), no. 2, 265-268.

[6] J. Meng, Y. B. Jun, and S. M. Hong, Implicative semilattices are equivalent to positive implicative BCK-algebras with condition (S), Math. Japon. 48 (1998), no. 2, 251-255.

Sergio Celani: Departamento de Matemática, Facultad de Ciencias Exactas, UniVerSidAd Nacional Del Centro, Pinto 399, 7000 TANDil, ARgentina

E-mail address: scelani@exa.unicen.edu.ar 


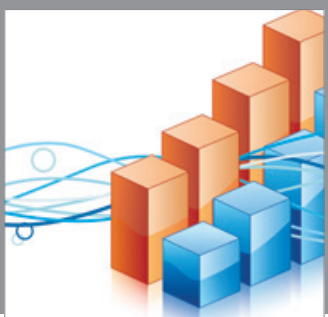

Advances in

Operations Research

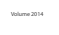

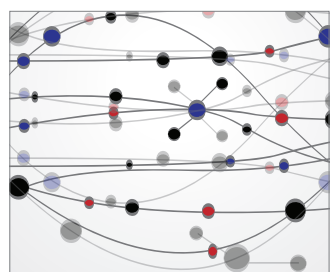

\section{The Scientific} World Journal
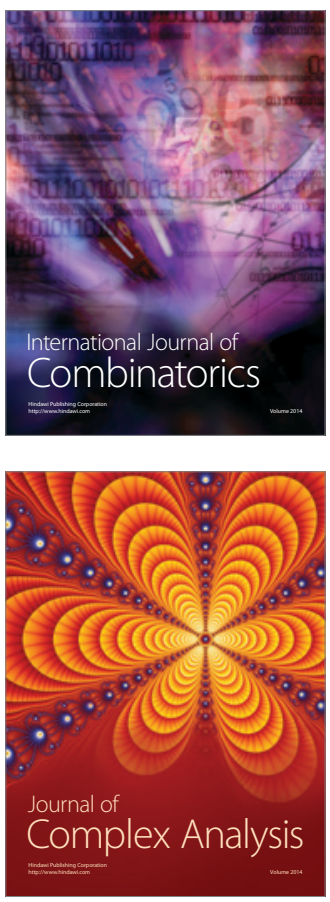

International Journal of

Mathematics and

Mathematical

Sciences
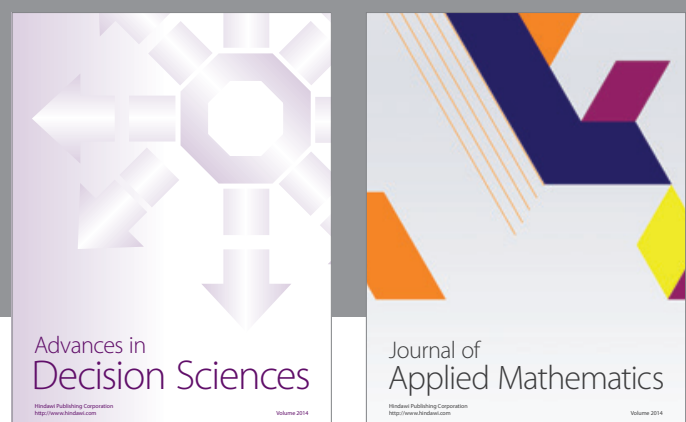

Journal of

Applied Mathematics
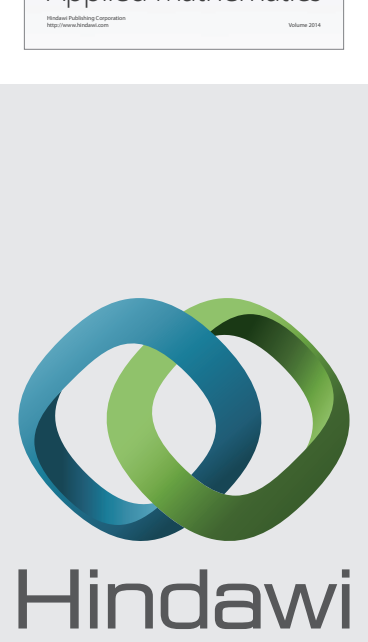

Submit your manuscripts at http://www.hindawi.com
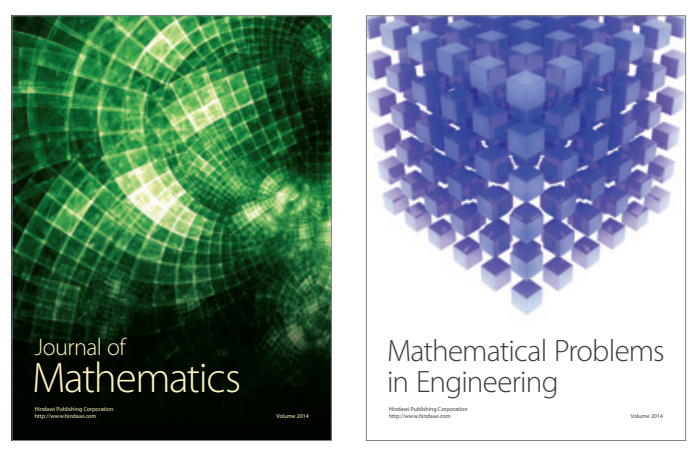

Mathematical Problems in Engineering
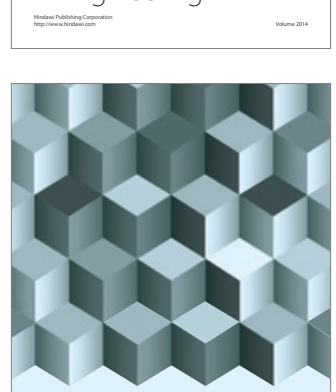

Journal of

Function Spaces
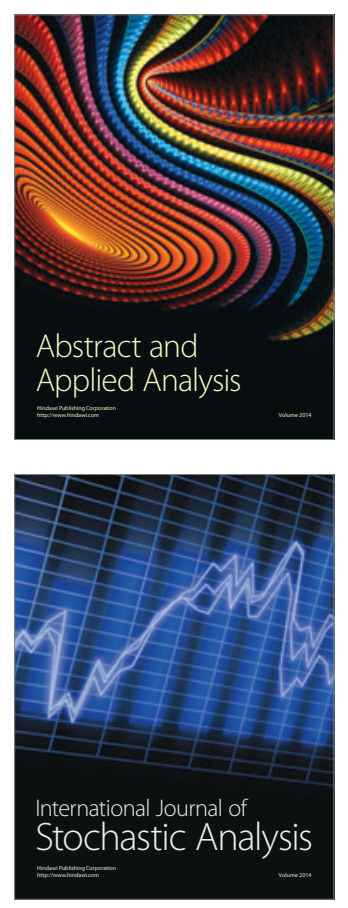

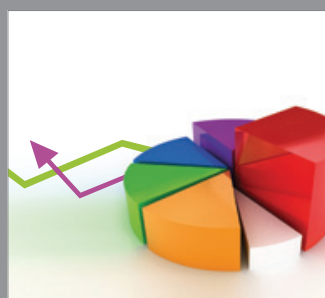

ournal of

Probability and Statistics

Promensencen
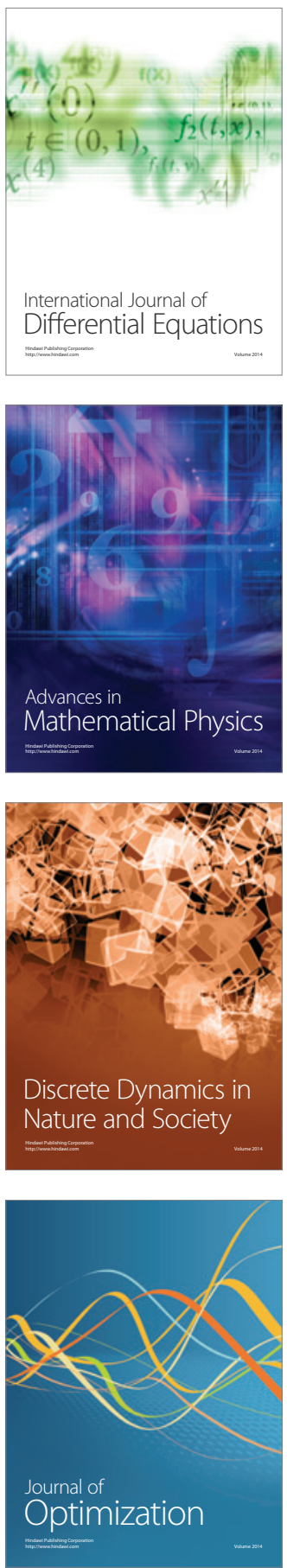